\title{
Occupational Blood Exposures in Health Care Workers at a Tertiary Teaching Hospital: Incidence, Characteristics and Transmission of Blood Borne Pathogens
}

\author{
Anitha Deva ${ }^{1 *}$ (D) , Prabhakar Kamarthi ${ }^{2}$ (D) Jebamani Hepzibai ${ }^{3}$ (D) , Jyothi A.V. ${ }^{3}$ (D) and \\ Beena Parvangada Madappa ${ }^{1}$ (D) \\ ${ }^{1}$ Department of Microbiology, ${ }^{2}$ Department of Medicine, ${ }^{3}$ Infection control nurse, SDUMC, SDUAHER, \\ Tamaka, Kolar, Karnataka, India.
}

\begin{abstract}
Health care workers (HCW) are at risk of contracting Hepatitis B, Hepatitis C and Human immunodeficiency viruses through Occupational Blood Exposure (OBE). Information on the incidence rate, epidemiological characteristics and transmission rate are essential to formulate effective preventive measures and management of OBE. From June-2016 to May-2018, OBE among HCW were captured systematically in EPINet (Exposure prevention information network) Register through Self reporting system. Data on number of in-patient days was collected. Incidence rate, epidemiological characteristics and transmission rate were analyzed and evaluated. Sixty seven OBE incidents were reported by HCWs accounting to an incidence rate of $0.2 / 1000$ in-patient days. Of these, $94 \%$ were Needle stick injuries and $6 \%$ were Blood and body fluid exposures. OBE incidents occurred mainly in wards, emergency department and operation theatre with a rate of $38.8 \%, 35.8 \%$ and $14.9 \%$ respectively. Exposures were encountered mainly by Interns (32.8\%), staff-nurses (22.4\%), Post-graduate students (14.9\%) and Nursing students (14.9\%). Recapping of the needle was the activity which led to OBE in $37.3 \%$ and improper disposal of sharps in $\mathbf{2 2 . 3 8} \%$ of the incidents. In addition, $\mathbf{1 9 . 4 \%}$ and $\mathbf{8 . 9 5 \%}$ of the incidents were due to accidental and patient factors respectively. None of the followed-up HCWs at the end of 6 months were infected by these exposures. The study brings out the epidemiological characteristics of $\mathrm{OBE}$ and throws a light on the target population among HCW who require more awareness and training to prevent OBE.
\end{abstract}

Keywords: Needle stick injury, Safe injection practices, Health care workers, Occupational blood exposures

*Correspondence: anithadeva77@gmail.com; 9663991461

(Received: September 19, 2019; accepted: February 05, 2020)

Citation: Anitha Deva, Prabhakar Kamarthi, Jebamani Hepzibai, Jyothi A.V. and Beena Parvangada Madappa, Occupational Blood Exposures in Health Care Workers at a Tertiary Teaching Hospital: Incidence, Characteristics and Transmission of Blood Borne Pathogens, J. Pure Appl. Microbiol., 2020; 14(1):609-614. https://doi.org/10.22207/JPAM.14.1.63

(C) The Author(s) 2020. Open Access. This article is distributed under the terms of the Creative Commons Attribution 4.0 International License which permits unrestricted use, sharing, distribution, and reproduction in any medium, provided you give appropriate credit to the original author(s) and the source, provide a link to the Creative Commons license, and indicate if changes were made. 


\section{INTRODUCTION}

Health care workers (HCW) are at increased risk of coming in contact with patients' blood and body fluids and thereby contracting blood borne pathogens. Many infections can get transmitted through Occupational blood exposure including, Hepatitis B virus (HBV), Hepatitis C virus (HCV) and Human immunodeficiency virus (HIV) infections ${ }^{1}$. An Occupational Blood Exposure (OBE) is a percutaneous injury (e.g. a needle stick injury or cut with a previously used sharp medical device) or contact between a mucous membrane or nonintact skin with blood, tissue, or other body fluids that may place a HCW at risk of HBV, HCV, or HIV infection $^{1}$. OBEs are common and to an extent inevitable in HCWs during patient care. According to WHO, out of 35 million HCWs worldwide, 3 million experience OBE. These exposures result in 70,000 HBV, 15,000 HCV and 500 HIV infections ${ }^{2}$. Due to $O B E$, the risk of transmission of infections range from as low as $0.2-0.5 \%$ for HIV to as high as $3-10 \%$ for $\mathrm{HCV}$ and $30 \%$ for $\mathrm{HBV}^{3,4}$. More than $90 \%$ of these infections occur in developing countries ${ }^{2}$. Also, OBEs result in significant psychological stress, depression and management costs 5 .

Though health care organizations have protocols to manage OBEs, OBE rates which reflect the lacunae in preventive protocols and negligence among HCWs and OBE associated infections in $\mathrm{HCW}$ are poorly evaluated ${ }^{6}$. All healthcare institutions should ensure that $\mathrm{HCW}$ receive training to recognize and report occupational blood exposures. It should have a system in place to facilitate reporting such as designating a clinical area for reporting, wherein the working system is the same for 24 hours and which is readily accessible, ideally the emergency department. Also, the postexposure assessment process and prophylaxis should readily be available in such areas for timely management of the $\mathrm{HCW}^{7}$. Information on the incidence rate, epidemiological characteristics and transmission rates are essential to formulate effective preventive measures like adhering to universal precautions, safe injection practices and immunization against Hepatitis $B$. Very few studies have been done on OBE in developing countries and there is no data available in our region ${ }^{3}$. The aim of this study is to determine the incidence rate and epidemiological characteristics of OBE among HCWs and evaluate the transmission rate of HBV, HIV and HCV due to these exposures.

\section{MATERIALS AND METHODS}

This observational prospective study was conducted at R.L. Jalappa hospital and research centre in southern India which is a tertiary care teaching hospital. It is a 1000 bedded tertiary care hospital with about 1100 - 1200 Health care workers at any given point of time involved in direct patient care; 200 consultants, 150 interns, 130 post graduate students, 590 nurses and nursing students, 82 housekeeping staff and 45 laboratory technicians.

Over a period of 2 years from June 1, 2016 to May 31, 2018, occupational blood exposures in health care workers were captured regularly and systematically in the EPINet (Exposure prevention information network) register through self-reporting system as per the Hospital infection control committee protocol 7,8 . According to the protocol, the exposed HCW reported to the physician on duty in the Emergency department as early as possible after the exposure, after taking the first aid measures. Serum samples from such exposed HCWs and the source patient (if the source patient was known) were drawn and sent to the laboratory immediately for HIV and HBsAg testing. Testing for HIV 1 and 2 was done by three different methods; Chemiluminescence assay, HIV Tridot and Aspen test, and reported as per the NACO guidelines ${ }^{9}$. HBsAg for Hepatitis $B$ infection was tested by chemiluminescence immunoassay and immunochromatography. The OBE incident report was obtained from the exposed HCW by the infection control team which included the following; demographic details of the HCW, designation of the HCW, place of incidence, activity which led to incident, type of injury, severity of injury, site of injury, hepatitis B immunization status of the HCW, immediate first aid measures taken like washing the injured area and the HIV and Hepatitis B status of the source patient if known. Data on the number of Health care workers in the hospital was obtained and the number of in-patient days on monthly basis were collected from the Tracking registers from all the patient care areas in the hospital. HCWs who got exposed to HIV seropositive patients were given a stat dose of Tenofovir 300mg + Lamivudine 
$300 \mathrm{mg}+$ Efavirenz 600mg as antiretroviral therapy (ART) in the Emergency department as per the instruction by the physician and further referred to the integrated testing and counselling centre (ICTC) attached to our hospital where they were treated with Tenofovir 300mg + Lamivudine 300mg + Efavirenz 600mg ART drugs for a period of 28 days. For follow-up, all HCWs were counselled and advised to get tested after 6 weeks, 3 months, and finally after 6 months. The outcome of such exposed HCWs was recorded.

\section{Statistical analysis}

All data were entered into Microsoft excel using SPSS statistical software (version 22). Incidence rate of OBE in a month was calculated by the formula ${ }^{8}$.

No.of OBEs in a month $\div$ No.of inpatient days in a month $X 1000$

The overall incidence over a period of 2 years was calculated by summating the monthly data. Epidemiological characteristics were analysed and evaluated.

\section{RESULTS}

There were a total of $67 \mathrm{OBE}$ incidents reported by health care workers and 3,35,000 inpatient days at R.L. Jalappa hospital and research centre, over a period of 2 years from June-2016 to May-2018 accounting to an OBE incidence rate of $0.2 / 1000$ in-patient days. Of these, $63(94 \%)$ exposures were Needle stick injuries or sharp injuries and $4(6 \%)$ were Blood and body fluid exposures.

With regard to the clinical areas, OBE incidents occurred mainly in wards and Emergency department. In the wards, there were 26 incidents accounting to a rate of $38.8 \%$ and in the emergency department, there were 24 incidents with a rate

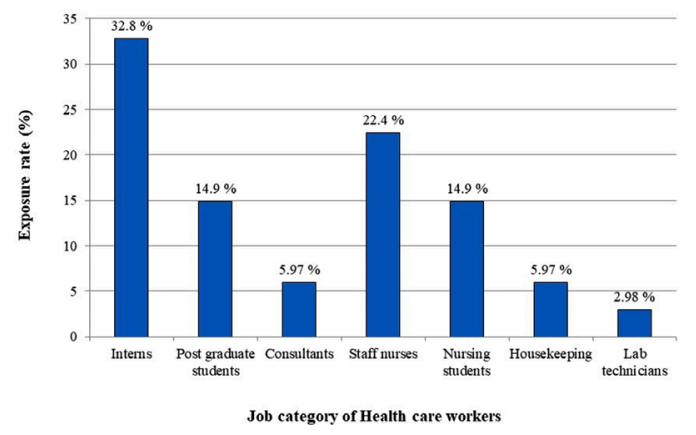

Fig. 1. Job category of health care workers who had OBE
Table 1. Clinical areas Where OBE occurred

\begin{tabular}{lc}
\hline $\begin{array}{l}\text { Clinical area where } \\
\text { incident occurred }\end{array}$ & $\mathrm{n} / \%$ \\
\hline Wards & $26(38.8)$ \\
Emergency department & $24(35.8)$ \\
Operation theatre & $10(14.92)$ \\
Paediatric ICU & $03(4.47)$ \\
Blood bank & $02(2.98)$ \\
Adult ICU & $01(1.5)$ \\
Dialysis unit & $01(1.5)$ \\
Total & $67(100 \%)$
\end{tabular}

of $35.8 \%$. Ten exposures were reported from operation theatre contributing for $14.9 \%$ of incidents. Different clinical areas where the OBE incidents occurred is depicted in Table 1. Other areas which had OBE incidents are pediatric ICU, blood bank, adult ICU and dialysis unit with rates of $4.47 \%, 2.98 \%, 1.5 \%$ and $1.5 \%$ respectively.

OBE among different category of HCWs is represented in Fig. 1. Exposures were encountered mainly by doctors (36/67, 53.73\%); Interns, Postgraduate students and consultants had $32.8 \%$, $14.9 \%$ and $5.97 \%$ of the exposures respectively. The incidents among the nurses accounted for $37.3 \%$ (25/67 incidents); $22.4 \%$ of the incidents occurred among staff nurses and $14.9 \%$ of the incidents occurred in nursing students. Unfortunately, housekeeping staff who are not directly involved in patient care, also got exposed due to improper sharp disposal methods by other HCWs and accounted for $5.97 \%$ (4/67) of the incidents. The least rate of $2.98 \%$ (2/67 incidents) was seen among laboratory technicians.

Exposure rate based on the incidents among different category of health care workers based on the total number of workers in each category is shown in Fig. 2. Exposure rate was $3.75 \%$ (36/960) , $2.59 \%$ (15/580), 2.44\% (4/164), $2.22 \%(2 / 90)$ and $1.67 \%(10 / 600)$ among doctors, staff nurses, house keepers, laboratory technicians and nursing students respectively.

The activity leading to the incident or the cause for these exposures is shown in Fig. 3. Recapping of the needle was the most common activity which led to OBE in $37.3 \%$ of the incidents followed by improper sharp disposal methods in $22.38 \%$ of the incidents. It was found that inadequate skills were the cause for the incidents 
in $11.94 \%$ of the exposures. To highlight the fact that exposures are inevitable at times in spite of effective training of health care workers and following vigilant practices, there were $19.4 \%$ and $8.95 \%$ of the incidents due to accidental and patient factors respectively.

All exposed health care workers were managed as per the NACO guidelines. Among 67 exposures, the source patients were positive for HIV and HBsAg in 4 cases each and source was unknown in 1 case. In 4/67 incidents, where the source patients were positive for HIV and in 1 incident, where the source was unknown, the healthcare workers were treated with antiretroviral drugs for a period of 28 days with counselling and monitoring. In other $4 / 67$ incidents, where the source patients were positive for $\mathrm{HBsAg}$, the exposed health care workers were tested for the anti HBs antibody titres. Three HCWs had protective Anti HBs titres whereas only $1 \mathrm{HCW}$ had inadequate antibody titres; this health care worker was administered Hepatitis B immunoglobulin within 48 hours of exposure and Hepatitis B vaccine series was started and

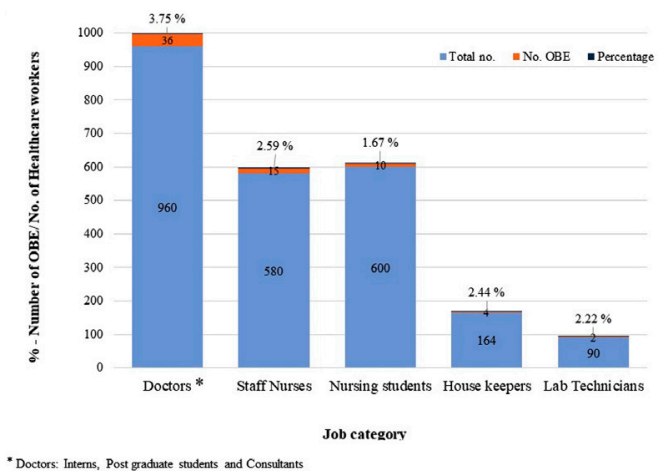

Fig. 2. Exposure rate among different catetgory of health care workers

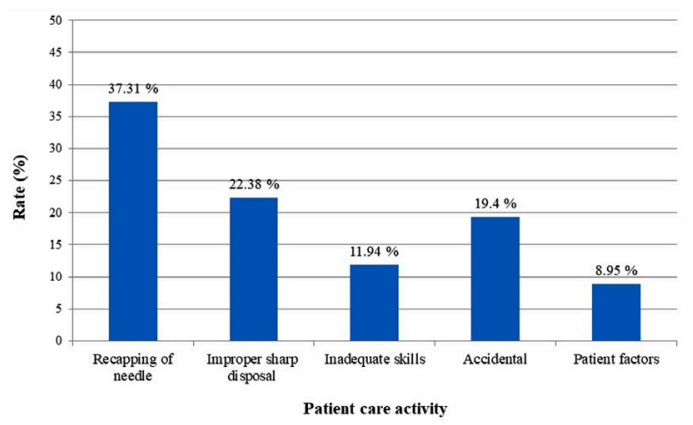

Fig. 3. Health care activity leading to OBE completed. Overall, 61 individuals did not require any Post exposure prophylaxis.

Out of 67 exposed HCWs, 15 were lost for follow up. Remaining 52 exposed HCWs were tested for HIV and HBsAg after 6 weeks, 3 months and finally at the end of 6 months after the incident. None of these followed up HCWs were infected by these exposures.

\section{DISCUSSION}

Occupational blood exposures in the form of sharp injuries and splashes of blood and body fluids are common among health-care workers which make them vulnerable for developing many blood-borne infectious diseases. Occupational exposures are common in the developing countries and it is believed that $40-75 \%$ of these exposures are not reported. Unreported exposures are a serious problem; they lead to HCWs not receiving post exposure prophylaxis (PEP) against HIV and hepatitis $B$ placing them at risk of developing these infections ${ }^{10}$.

Our study found an OBE incidence rate of $0.2 / 1000$ inpatient-days. The incidence is less when compared to other studies ${ }^{11}$. Regular induction training and periodic training of all category of health care workers on occupational blood exposures and the importance of its reporting and management led to good reporting system in our study as well as helped to achieve a low incidence rate.

With regard to the clinical areas, incidents occurred mainly in wards (38.8\%) and Emergency department (35.8\%) followed by Operation theatre (14.92\%). This is similar to the findings in a study done by Lee et al. ${ }^{6}$

Among job categories, exposures were encountered mainly by Doctors who contributed for $53.73 \%$ of the incidents, followed by staffnurses $(22.4 \%)$ and others which is in concordance with other studies ${ }^{3,12}$. Among doctors, interns and post graduate students accounted for majority of the incidents which corresponds with other reports from India ${ }^{13,14}$. This is probably because they are more commonly involved in clinical procedures in our set up as it is a teaching hospital. Most of the exposures occurred in newly joined interns and post graduate students. This can be attributed to factors like, lack of experience, less knowledge regarding safe injection practices and 
handling the heavy workload of patients under pressure situations.

With respect to the health care activities leading to OBE, accidental events, patient factors and inadequate skills together accounted for the majority of incidents $(40.3 \%)$ which are very difficult to prevent. Although, it may not be practically feasible to avoid their occurrence altogether, it can be minimized to a large extent by following safe practices. Recapping of needle and improper disposal of needle accounted for $37.31 \%$ and $22.38 \%$ of the OBE incidents respectively and similar findings were reported by a study at Delhi ${ }^{15}$. Even though, the contaminated needles and other contaminated sharps should not be bent, recapped, or removed, many studies have revealed that recapping is still prevalent among HCWs which also holds good in this study ${ }^{11,15}$. To emphasise on these preventable aspects of OBEs and the importance of OBE reporting, our hospital has implemented induction training for new HCWs and annual continuing education for all HCWs. The present study did not show any seroconversion in HCWs who could be followed up after OBE. Our hospital's strict infection control policy being in place with timely management of the exposed HCWs as per guidelines, likely contributed to achieve this. Although, there was no transmission in these HCWs, this could be finally known only after 6 months of the OBE incident. Therefore, after the incident and during the period of followup, HCWs went through severe psychological stress anticipating negative consequences. Few studies have shown the transmission in exposed individuals ${ }^{6}$.

\section{CONCLUSION}

The study brings out the epidemiological characteristics of exposed health care workers, thereby throwing a light on the target population who require more awareness and training to prevent OBE. Every effort should be made to prevent OBE through education and implementation of safe injection and working practices. Providing initial and continuing training for health care workers is very important. Prevention of OBE by adhering to the universal precautions, must remain a priority in any healthcare setting to prevent the transmission of HIV, Hepatitis B and $\mathrm{HCV}$ as well as to avoid the psychological trauma.

\section{ACKNOWLEDGMENTS}

We would like to express our heartfelt thanks to Dr. Mohan Kumar, and Mrs. Beenamma, RLJH \& RC for providing assistance on Protocols and Support.

\section{CONFLICT OF INTEREST}

The authors declare that there is no conflict of interest.

\section{FUNDING}

None.

\section{AUTHORS' CONTRIBUTIONS}

All authors listed have made a substantial, direct and intellectual contribution to the work, and approved it for publication.

\section{DATA AVAILABILITY}

All datasets generated or analyzed during this study are included in the manuscript.

\section{ETHICS STATEMENT}

The study was approved by the institutional Ethics committee (IEC).

\section{REFERENCES}

1. Centers for Disease Control and Prevention: NIOSH Publications and Products. Stop sticks: Stop injuries. Available from: https://www.cdc.gov/niosh/stopsticks/ sharpsinjuries.html

2. World Health Organization-WHO, "Occupational health, needlestick injuries," 2016, http://www. who.int/occupational_health/topics/needinjuries/ en/. View at Google Scholar

3. Goel V, Kumar D, Lingaiah R, Singh S. Occurrence of Needlestick Injuries among Health-care Workers of a Tertiary Care Teaching Hospital in North India. Journal of Laboratory Physicians, 2017; 9: 20-25. https://doi. org/10.4103/0974-2727.187917

4. Cheng HC, Su CY, Yen AM, Huang CF. Factors affecting occupational exposure to needlestick and sharps injuries among dentists in Taiwan: A nationwide survey. PLoS One, 2012; 7: e34911. https://doi. org/10.1371/journal.pone.0034911

5. Sohn J-W, Kim B-G, Kim S-H, Han C. Mental health of healthcare workers who experience needlestick and sharps injuries. J Occup Health, 2006; 48: 474-9. https://doi.org/10.1539/joh.48.474

6. Lee JH, Cho J, Kim YJ, Im SH, Jang ES, Kim JW etal. Occupational blood exposures in health care workers: incidence, characteristics, and transmission of blood borne pathogens in South Korea. BMC Public Health, 2017; 17: 827. https://doi.org/10.1186/s12889-0174844-0 
7. Centers for Disease Control and Prevention. CDC Guidance for Evaluating Health-Care Personnel for Hepatitis B Virus Protection and for Administering Postexposure Management. MMWR, 2013; 62: 2.

8. EPINet Report for Needlestick and Sharp Object Injuries. at https:// international safety center.org/ exposure-reports/.

9. Ministry of Health and Family Welfare. National AIDS Control Organization: National Guidelines for HIV Testing, 2015.

10. Wilburn SQ. Needlestick and sharps injury prevention. Online J Issues Nurs, 2004; 9: 5.

11. International Healthcare Worker Safety Center, University of Virginia. U.S. EPINet Sharps Injury and Blood and Body Fluid Exposure Surveillance Research Group. Sharps Injury Data Report. 2011. [Last accessed on 2015 Jun 11]. Available from: http:// www.healthsystem.virginia.edu/pub/epinet/ epinetdatareports.html\#CitingEPINet.
12. Holla R, Unnikrishnan B, Ram P, Thapar R, Mithra P, et al. Occupational Exposure to Needle Stick Injuries among Health Care Personnel in a Tertiary Care Hospital: A Cross Sectional Study. J Community Med Health Educ, 2014 S2: 004. https://doi.org/10.4172/2161-0711. S2-004

13. Sharma A, Gur R, Bhalla P. Study on prevalence of needle stick injury among health care workers in a tertiary care hospital in New Delhi: A two-year review. Indian J Public Health, 2012; 56: 101-3. https:// doi.org/10.4103/0019-557X.96987

14. Prakash KP. Epidemiology of needle-stick injuries in Mangalore. J Evol Med Dent Sci, 2012; 1: 128-36. https://doi.org/10.14260/jemds/22

15. Sharma R, Rasania SK, Verma A, Singh S. Study of Prevalence and Response to Needle Stick Injuries among Health Care Workers in a Tertiary Care Hospital in Delhi, India. Indian J Community Med, 2010; 35: 74-77. https://doi.org/10.4103/0970-0218.62565 